\title{
The Promise of next generation sequencing micro RNA for the discovery of new targets in contrast induced acute kidney injury
}

\author{
Ayman Haq ${ }^{1}$, Peter A. McCullough ${ }^{1,2,3}$ \\ ${ }^{1}$ Department of Internal Medicine, Baylor University Medical Center, Dallas, TX, USA; ${ }^{2}$ Baylor Jack and Jane Hamilton Heart and Vascular \\ Hospital, Dallas, TX, USA; ${ }^{3}$ Baylor Heart and Vascular Institute, Dallas, TX, USA \\ Correspondence to: Peter A. McCullough, MD, MPH. Baylor Heart and Vascular Institute, 621 N. Hall St, H030, Dallas, TX 75226, USA. \\ Email: peteramccullough@gmail.com. \\ Comment on: Liu Y, Liu B, Liu Y, et al. MicroRNA expression profile by next-generation sequencing in a novel rat model of contrast-induced acute \\ kidney injury. Ann Transl Med 2019;7:178.
}

Submitted Jul 17, 2019. Accepted for publication Jul 25, 2019.

doi: $10.21037 / \mathrm{atm} .2019 .07 .83$

View this article at: http://dx.doi.org/10.21037/atm.2019.07.83

Contrast induced acute kidney injury (CI-AKI) is defined by KDIGO as an increase in serum creatinine by 1.5 times within 7 days of exposure to contrast, a serum creatinine increase of $0.3 \mathrm{mg} / \mathrm{dL}$ or a urine output less than $0.5 \mathrm{~mL} / \mathrm{kg}$ of body weight for at least $6 \mathrm{~h}$ following iodinated contrast exposure (1). Patients with preexisting renal disease are at greatest risk for developing CI-AKI. Its pathogenesis is thought to be multifactorial. Contrast initially causes nitric oxide mediated vasodilation of renal vasculature. This is followed by intrarenal vasoconstriction that persists for several hours causing ischemia, particularly in the outer medulla where the oxygen tension is lower than that of many tissues (2). As it is water soluble, contrast is freely filtered by the glomerulus with little alteration of podocyte or filtration barrier function. However, it is taken up by proximal tubular cells and results in cellular dysfunction and apoptosis. The contrast also permeates into the tubulointerstitial space leading to the loss of cellular architecture. Tubular epithelial cells slough off into the tubular space, thus reducing the clearance of contrast and promoting its deleterious effects. The cellular damage also results in the formation of reactive oxygen species which perpetuate cellular injury. Since the glomerulus is relatively unaffected, the reduction in glomerular filtration and rise in serum creatinine associated with CI-AKI is mediated by tubuloglomerular feedback and occurs well after the renal parenchyma has been injured (3). However, the exact mechanism of CI-AKI remains unclear and no definitive therapy has been established.
Micro RNA (miRNA) were discovered in 1993 when investigating the larval development of Caenorbabditis elegans. The decades have since shown that miRNA is a vital post-transcriptional regulatory mechanism of gene expression. They are short, 22 nucleotides in length, and are derived from non-coding regions of DNA. While alterations of DNA chromatin structure serve to up or down regulate gene expression, miRNA primarily downregulates gene expression. RNA polymerase II initially transcribes large 'pri-miRNA' hairpins which are processed by the Drosha/DGCR8 complex to produce smaller 'pre-miRNA' hairpins. These are then transported to the cytoplasm and the endonuclease Dicer cuts the premiRNA near the loop of the hairpin to produce the mature miRNA duplex. This duplex is loaded onto Argonaute and the expulsion of one of the duplex strands forms the RNAinduced silencing complex (RISC). The RISC binds the three prime untranslated region of messenger RNA (mRNA) at the target binding site and recruits a series of factors that suppress the translation and promote the degradation of mRNA transcripts. Each miRNA can potentially bind to hundreds of unique mRNA, as mRNAs are under selective pressure to conserve their target binding sites (4). The miRNA seed sequence, a 6- 8 nucleotide region, determines which mRNA target binding site the miRNA will be receptive to. For example, the seed sequence of miR-200a and miR-141 (AACACU) differs by the seed sequence of miR-200b, miR-200c, and miR-429 (AAUACU) by one nucleotide, conferring different target binding sites. 
However, since mRNA can have more than one target binding site, these seed sequences bind different targets on the same mRNA. Hence, these 5 miRNA can be classified into one family (5). From a clinical and research perspective, kidney diseases offer a unique opportunity to assay both the plasma and urine for miRNA and along with that, many challenges in execution and interpretation (Table 1).

In the present study Liu and colleagues mapped the miRNA profile of CI-AKI model. Male Sprague-Dawley rats were anesthetized with $10 \%$ chloral hydrate $(15 \mathrm{~mL} / \mathrm{kg})$ and subsequently administered furosemide $(15 \mathrm{~mL} / \mathrm{kg})$ intraperitoneally. After $6 \mathrm{~h}$ of volume depletion, the rats were injected with iopromide contrast (Ultravist, $15 \mathrm{~mL} / \mathrm{kg}$ ) via their tail veins, and another $6 \mathrm{~h}$ of dehydration was sustained. The control was injected with saline instead of contrast. Serum creatinine, blood urea nitrogen (BUN), cystatin $\mathrm{C}$, neutrophil gelatinase-associated lipocalin, and kidney injury molecule 1 concentrations were measured at baseline and $24 \mathrm{~h}$ after contrast administration. They were all significantly elevated in the CI-AKI group and unchanged in the control group.

The rats were then sacrificed, and their kidneys were harvested and histologically examined. Total RNA was isolated and treated with DNase I and then enriched for mRNA with oligo (dT) magnetic beads. The mRNA was used to created double-stranded cDNA, which was amplified and sequenced by Illumina HiSeq ${ }^{\text {TM }} 2000$ and analyzed by Illumina Genome Analyzer II. These reads were filtered for quality with a false discovery rate (FDR) of $\leq 0.001$ and non-miRNA sequences were identified and excluded. Known miRNA and potential miRNA with edited seed sequences were identified, with 100 that were upregulated and 107 that were downregulated in the CIAKI group. The criterion for differential gene expression was set at two-fold or greater $(\log 2$-ratio $\geq 1)$ and 19 significantly upregulated and 22 significantly downregulated miRNA were identified. Potential targets of these miRNA were identified by miRanda and RNAhybrid and mapped to cellular pathways, including those of inflammation, endoplasmic reticular stress, and apoptosis.

For verification, RNA samples were independently collected and 13 of the differentially expressed miRNA were examined by quantitative real-time polymerase chain reaction (qRT-PCR). U6 was employed as a control for miRNA and the $2^{-\Delta \Delta \mathrm{Ct}}$ method was used to determine relative gene expression.

We considered the three miRNA that showed the greatest upregulation and downregulation as they were least likely to be 'bystanders' (4). These were miR-3558-5p ( 30fold increase), miR-34c-3p ( 13.5-fold increase), miR-384$5 \mathrm{p}$ ( 9.5-fold increase), miR-31a-5p ( 4-fold decrease), miR-328a-5p ( 13-fold decrease, miR-377-3p ( 41-fold decrease). A brief literature search reveals that these miRNA (excluding miR-3558-5p) have been associated with tumour suppressive functions in various cancers, except for miR31 , which has been more strongly associated with tumour promotion (18-22). The lack of kidney specific functions led us to believe these signals are "reporting the news" and may not be playing a role in either pathophysiology or adaptive cellular protection.

Furthermore, determining the relevant mRNA targets of the differentially expressed miRNA is another quandary. An analysis of miR-233 targets in phenotypically normal mice revealed that 150 of 192 examined targets had greater variability between individual mice than the median magnitude of miR-223 mediated suppression. Thus, these targets were unlikely to be significantly suppressed by miR233. Since, miRNA mediated suppression itself is moderate, only a subset of the remaining targets would be sensitive enough to miRNA suppression to produce phenotypic change (4). These 'sensitive targets' would be among the most highly conserved. However, some highly conserved targets may exist to 'soak-up' miRNA and prevent the over-suppression of sensitive targets. Other reasons for target conservation besides direct phenotypic change likely exist as well, hence, current computational miRNA target prediction methods are insufficient to delineate relevant miRNA targets (17).

Similar analyses of renal tissue by microarray assay were recently performed by Gutiérrez-Escolano and colleagues who found 17 differentially expressed miRNA and Sun and colleagues who found 11 differentially expressed miRNA $(12,13)$. Both the present study and Gutiérrez-Escolano et al. found downregulation of miR-24 and miR-200 isoforms. However, there was no agreement in upregulated miRNA. Furthermore, the present authors found upregulation of let-7a and let-7f, while Gutierrez-Escolano et al. found downregulation of let-7c, an isoform with an identical seed sequence (23). The report by Sun et al. is similarly incongruent with the present study, with upregulation of let-7b being the only common miRNA. A brief comparison of the findings of Gutiérrez-Escolano et al. and Sun et al. reinforces the lack of reproducibility with only two miRNA families (miR-30 and let-7) in common. A 2016 review of miRNA in AKI is similarly disappointing since many of the most extensively studied miRNA (miR-210, miR-21, miR- 
Table 1 Sources of error in miRNA profiling with NGS in the CI-AKI model

\begin{tabular}{|c|c|c|c|}
\hline Source of error & Mechanism & Proposed solution & References \\
\hline TRIzol reagent & $\begin{array}{l}\text { Degradation of certain miRNAs during the RNA } \\
\text { extraction process }\end{array}$ & $\begin{array}{l}\text { Employ alternative reagents in the RNA extraction } \\
\text { process }\end{array}$ & (8) \\
\hline $\begin{array}{l}\text { High sensitivity of } \\
\text { NGS }\end{array}$ & $\begin{array}{l}\text { NGS has increased rates of type } 1 \text { error } \\
\text { compared to conventional methods }\end{array}$ & $\begin{array}{l}\text { Employ more conservative } p \text {-values for a stringent } \\
\text { FDR }\end{array}$ & (7) \\
\hline $\begin{array}{l}\text { qRT-PCR is a } \\
\text { suboptimal control for }\end{array}$ & Variable U6 expression & $\begin{array}{l}\text { Employ an internal control with less variable } \\
\text { expression }\end{array}$ & $(10,11)$ \\
\hline NGS & $\begin{array}{l}2^{-\Delta \Delta C t} \text { method assumes perfectly efficient PCR } \\
\text { Variable background fluorescence can } \\
\text { confound result }\end{array}$ & $\begin{array}{l}\text { Compensate for PCR inefficiency and variable } \\
\text { background fluorescence with a modified } \\
\text { algorithm, such as the one proposed by Rao et al. }\end{array}$ & \\
\hline miRNA nomenclature & $\begin{array}{l}\text { miRNAs are named in the sequential order of } \\
\text { their discovery, not for their seed sequences }\end{array}$ & $\begin{array}{l}\text { Maintain strict nomenclature, including } \\
\text { annotation for isoforms and } 3 p / 5 p \text { strands }\end{array}$ & (4) \\
\hline & $\begin{array}{l}\text { The } 5 p \text { and } 3 p \text { strands can have different } \\
\text { targets }\end{array}$ & $\begin{array}{l}\text { Identify the seed sequence and targets for } \\
\text { miRNAs of interest }\end{array}$ & \\
\hline $\begin{array}{l}\text { Short length and high } \\
\text { homology of miRNAs }\end{array}$ & $\begin{array}{l}\text { Random errors of only a few nucleotides can } \\
\text { cause the misidentification of the miRNA }\end{array}$ & $\begin{array}{l}\text { Larger sample sizes and multiple assays with } \\
\text { averaging will minimize this random error }\end{array}$ & (7) \\
\hline $\begin{array}{l}\text { Computational miRNA } \\
\text { target prediction }\end{array}$ & $\begin{array}{l}\text { Many, if not most, conserved miRNA targets } \\
\text { undergo mild suppression without associated } \\
\text { phenotypic change }\end{array}$ & $\begin{array}{l}\text { Confirm target predictions with selective } \\
\text { knock-out and overexpression studies of both the } \\
\text { miRNA of interest and its predicted targets to } \\
\text { confirm phenotypic correlation }\end{array}$ & (17) \\
\hline $\begin{array}{l}\text { Extra-renal miRNA in } \\
\text { renal tissue }\end{array}$ & $\begin{array}{l}\text { miRNA in serum may undergo renal elimination } \\
\text { and be inappropriately detected within renal } \\
\text { tissue }\end{array}$ & $\begin{array}{l}\text { Simultaneous measurement of serum and renal } \\
\text { miRNA expression } \\
\text { Compare in vitro and ex vivo Cl-AKI miRNA } \\
\text { expression }\end{array}$ & (14) \\
\hline
\end{tabular}

Cl-AKI, contrast induced acute kidney injury; miRNA, micro RNA; NGS, next generation sequencing; FDR, false discovery rate; qRT-PCR, quantitative real-time polymerase chain reaction; PCR, polymerase chain reaction. 
320 , miR-192, etc.) have been found to be upregulated in some studies and downregulated in others (6). Hence, there is an immediate issue of a lack of concordance in both miRNA and the direction of up/down regulation in similar models of AKI from one lab to another.

The lack of reproducibility is not isolated to CI-AKI models. A review of miRNA in coronary artery disease found 29 studies, with the majority contradicting each other (7). Part of the incongruency can be attributed to next generation sequencing (NGS). Current adapter ligation methods can introduce bias into library preparation, which will skew miRNA detection compared to microarray assay. NGS is also more sensitive than microarray assay and will detect a larger number of 'bystander' miRNA. This is evident as the present study detected more than twice the differentially expressed miRNA as compared to GutiérrezEscolano and Sun et al. Additionally, TRIzol reagent, which was used in all three studies, can degrade certain miRNAs during the extraction process leading to further bias in library preparation $(8,9)$.

miRNA themselves present unique challenges to highthroughput sequencing. They are short in length and have a high degree of homology, sometimes differing by only a few nucleotides. Undetected errors early in the sequencing process can result in the misidentification of miRNA (7). Conversely, some miRNA seed sequences may be more closely related that their name suggests, as is the case of the miR-200 family. Hence, there may be a higher degree of congruency than initially evident due to evolving nomenclature.

In the present study Liu and colleagues do not indicate a sample size nor do they report observed power for the main hypothesis being tested, however it is fair to mention that small sample sizes were used by Gutiérrez-Escolano and Sun et al. at 9 and 12 animals respectively. Small sample sizes have been shown to dramatically increase the rate of false-positives and false-negatives in high-throughput miRNA sequencing (7). While qRT-PCR was used to verify the NGS and microarray assay results, there is evidence that this may be suboptimal as well $(10,11)$. Finally, the p-value assigned to the FDR can vary from study to study, and hence miRNA that would be screened out in one study could be reported as a significant finding in another study. Hence, at this early stage it is prudent to select more conservative p-values to guard against Type 1 error and thus, have fewer candidates move forward as potential signals of disease. Different experimental models invariably contribute to heterogeneity and external incongruency.
Gutiérrez-Escolano et al. found upregulation of serum miR-320 in their mouse model, but not in their human case-control analysis. Furthermore, there is evidence that miRNAs circulating in serum are renally eliminated (24). As dehydration and contrast administration have systemic sequela, some of the detected miRNAs may have extra-renal origins (3).

Lastly, miRNA expression in AKI is temporally dependent. Analysis of miRNA levels at a single point in time is insufficient in detecting differentially expressed miRNAs. Notably, miR-30 levels were shown to peak $4 \mathrm{~h}$ after CI-AKI and decline thereafter (13). The temporal rise and fall of certain miRNA may corresponding to vasoconstriction/ischemia, chemotoxicity, cell-cycle arrest, oxidative stress and cell to cell wavefront injury, recovery, and then ultimately tubule regeneration. Detailed studies are needed with long timelines after contrast exposure in order to be optimally informative about miRNA behavior in CI-AKI.

Gutierrez-Escolano et al. found significantly elevated levels of miR-30a, miR-30c, and miR-30e in the serum of CI-AKI mouse and human groups within 3-6 h of contrast administration. Receiver operating characteristic (ROC) analysis revealed specificity $>98 \%$ at a sensitivity of $85-60 \%$ at the optimal cutpoint. Sun et al. found similar results with significantly elevated levels of miR-30a and miR$30 \mathrm{e}$ in the serum of the CI-AKI mouse group 4-6 h of contrast administration. A follow-up case-control analysis of patients who received an elective cardiac angiogram and/ or PCI and developed CI-AKI confirmed elevated levels of miR-30a and miR-30e in the serum 4-6 h post-procedure with specificities $>93 \%$ at a sensitivity of $52-65 \%$. By comparison, the US Food and Drug Administration cleared NephroCheck ${ }^{\circledR}$ test used in critically ill patients has a sensitivity of $92 \%$ and specificity of $46 \%$ at the lower cutoff of 0.3 for AKI within $12 \mathrm{~h}$ (25). Hence, miR-30 and its isoforms have the potential to serve as highly specific adjunctive markers for the very early diagnosis for CI-AKI.

A series of studies highlight the potential for NGS in the progression of chronic kidney disease. Shi and colleagues reported that when Dicer was postnatally (postorganogenesis) deleted from mice renal podocytes, the mice developed early glomerulopathy, proteinuria, and end-stage renal disease (ESRD) at an average age of $\sim 8$ weeks (14). Postnatal deletion of Dicer from mice renal collecting ducts similarly produced tubulo-interstitial fibrosis, ESRD, and death by a median of 6 months (15). Yet, when Wei et al. postnatally deleted Dicer from mice renal proximal tubular 
cells these effects were not observed. Furthermore, after renal ischemia, these mice had lower serum creatinine and BUN, decreased renal cortical apoptosis, decreased tubular injury, and increased survival at 14 days compared to wild type animals (16). Renal ischemia is a mechanism of CI-AKI and renal proximal tubular cells play an intimate role in the pathogenesis of CI-AKI (3). Hence, these findings suggest that miRNA activity could be maladaptive at one time point and yet protective at another in models of CI-AKI.

In summary, miRNA signals detected by NGS techniques hold great promise for creating new knowledge and new insights into the pathogenesis of disease and recovery from illness. CI-AKI is a unique model since we understand the timing of injury. This is very important in the clinical development of both in vitro diagnostics and for pharmacotherapeutics. We look forward to much larger studies with more detailed measurement both in plasma and in urine with stringent false detection methods and detailed analyses of relevant miRNA targets. Something must be known about the function of candidate miRNA such as regulation of cell-cycle arrest, renal tubular cell stress, oxidative injury, apoptosis, autophagy, and recovery. We anticipate this approach will ultimately fulfill the promise of this breakthrough technology.

\section{Acknowledgments}

None.

\section{Footnote}

Conflicts of Interest: The authors have no conflicts of interest to declare.

Ethical Statement: The authors are accountable for all aspects of the work in ensuring that questions related to the accuracy or integrity of any part of the work are appropriately investigated and resolved.

\section{References}

1. Khwaja A. KDIGO clinical practice guidelines for acute kidney injury. Nephron Clin Pract 2012;120:c179-84.

2. McCullough PA. Radiocontrast-induced acute kidney injury. Nephron Physiol 2008;109:p61-72.

3. McCullough PA, Choi JP, Feghali GA, et al. ContrastInduced Acute Kidney Injury. J Am Coll Cardiol 2016;68:1465-73.
4. Bartel DP. Metazoan MicroRNAs. Cell 2018;173:20-51.

5. Korpal M, Lee ES, Hu G, et al. The miR-200 family inhibits epithelial-mesenchymal transition and cancer cell migration by direct targeting of E-cadherin transcriptional repressors ZEB1 and ZEB2. J Biol Chem 2008;283:14910-4.

6. Fan PC, Chen CC, Chen YC, et al. MicroRNAs in acute kidney injury. Hum Genomics 2016;10:29.

7. Kok MGM, de Ronde MWJ, Moerland PD, et al. Small sample sizes in high-throughput miRNA screens: A common pitfall for the identification of miRNA biomarkers. Biomol Detect Quantif 2017;15:1-5.

8. van Dijk EL, Jaszczyszyn Y, Thermes C. Library preparation methods for next-generation sequencing: tone down the bias. Exp Cell Res 2014;322:12-20.

9. Zhuang F, Fuchs RT, Robb GB. Small RNA expression profiling by high-throughput sequencing: implications of enzymatic manipulation. J Nucleic Acids 2012;2012:360358.

10. Rao X, Huang X, Zhou Z, et al. An improvement of the $2^{\wedge}$ (-delta delta CT) method for quantitative real- time polymerase chain reaction data analysis. Biostat Bioinforma Biomath 2013;3:71-85.

11. Xiang M, Zeng Y, Yang R, et al. U6 is not a suitable endogenous control for the quantification of circulating microRNAs. Biochem Biophys Res Commun 2014;454:210-4.

12. Gutiérrez-Escolano A, Santacruz-Vázquez E, GómezPérez F. Dysregulated microRNAs involved in contrastinduced acute kidney injury in rat and human. Ren Fail 2015;37:1498-506.

13. Sun SQ, Zhang T, Ding D, et al. Circulating MicroRNA-188, -30a, and -30e as Early Biomarkers for Contrast-Induced Acute Kidney Injury. J Am Heart Assoc 2016. doi: 10.1161/JAHA.116.004138.

14. Shi S, Yu L, Chiu C, et al. Podocyte-selective deletion of dicer induces proteinuria and glomerulosclerosis. J Am Soc Nephrol 2008;19:2159-69.

15. Hajarnis S, Yheskel M, Williams D, et al. Suppression of microRNA Activity in Kidney Collecting Ducts Induces Partial Loss of Epithelial Phenotype and Renal Fibrosis. J Am Soc Nephrol 2018;29:518-31.

16. Wei Q, Bhatt K, He HZ, et al. Targeted deletion of Dicer from proximal tubules protects against renal ischemiareperfusion injury. J Am Soc Nephrol 2010;21:756-61.

17. Pinzón N, Li B, Martinez L, et al. microRNA target prediction programs predict many false positives. Genome Res 2017;27:234-45.

18. Zhou YL, Xu YJ, Qiao CW. MiR-34c-3p suppresses the 
proliferation and invasion of non-small cell lung cancer (NSCLC) by inhibiting PAC1/MAPK pathway. Int J Clin Exp Pathol 2015;8:6312-22.

19. Song H, Rao Y, Zhang G, et al. MicroRNA-384 Inhibits the Growth and Invasion of Renal Cell Carcinoma Cells by Targeting Astrocyte Elevated Gene 1. Oncol Res 2018;26:457-66.

20. Yuan J, Zheng Z, Zheng Y, et al. microRNA-328 is a favorable prognostic marker in human glioma via suppressing invasive and proliferative phenotypes of malignant cells. Int J Neurosci 2016;126:145-53.

21. Azizi M, Fard-Esfahani P, Mahmoodzadeh H, et al. MiR377 reverses cancerous phenotypes of pancreatic cells via suppressing DNMT1 and demethylating tumor suppressor genes. Epigenomics 2017;9:1059-75.

22. Yu T, Ma P, Wu D, et al. Functions and mechanisms of

Cite this article as: Haq A, McCullough PA. The Promise of next generation sequencing micro RNA for the discovery of new targets in contrast induced acute kidney injury. Ann Transl Med 2019;7(18):424. doi: 10.21037/atm.2019.07.83
microRNA-31 in human cancers. Biomed Pharmacother 2018;108:1162-9.

23. Su JL, Chen PS, Johansson G, et al. Function and regulation of let-7 family microRNAs. Microrna 2012;1:34-9.

24. Gidlöf O, Andersson P, van der Pals J, et al. Cardiospecific microRNA plasma levels correlate with troponin and cardiac function in patients with ST elevation myocardial infarction, are selectively dependent on renal elimination, and can be detected in urine samples. Cardiology 2011;118:217-26.

25. Bihorac A, Chawla LS, Shaw AD, et al. Validation of cell-cycle arrest biomarkers for acute kidney injury using clinical adjudication. Am J Respir Crit Care Med 2014;189:932-9. 